\title{
Tissue-specific therapy in immune-mediated kidney diseases: new ARGuments for targeting the IL-23/IL-17 axis
}

\author{
Christian F. Krebs,, ${ }^{1,2,3}$ Jan-Eric Turner, ${ }^{2,3}$ Jan-Hendrik Riedel, ${ }^{1,2,3}$ and UIf Panzer,2,3 \\ 'Division of Translational Immunology, III. Department of Medicine, ${ }^{2 H a m b u r g ~ C e n t e r ~ f o r ~ T r a n s l a t i o n a l ~ I m m u n o l o g y ~(H C T I), ~ a n d ~} 3$ III. Department of Medicine, University Medical Center Hamburg-Eppendorf, \\ Hamburg, Germany.
}

\begin{abstract}
Immune-mediated kidney diseases are a leading cause of end-stage renal disease. Despite recent discoveries, the immunopathogenesis of this heterogeneous disease group remains incompletely understood, which is a major reason for the lack of specific therapies and targeted interventions. Accumulating evidence suggests that cytokines related to the $T$ cell response play an important role in renal autoimmunity. In this issue of the $J C l$, Li et al. demonstrate that IL-23 directly regulates the metabolism of parenchymal kidney cells, thereby generating a proinflammatory microenvironment that exacerbates $\mathrm{T}$ cell-driven renal tissue damage. These findings identify the IL23/IL-17 axis as a key mediator of renal tissue injury and open new avenues for the development of pathogenesis-based treatment strategies in renal inflammatory diseases.
\end{abstract}

\section{Immune-mediated kidney diseases}

Immune-mediated kidney injury can be a consequence of systemic diseases, such as anti-neutrophil cytoplasmic antibody-associated (ANCA-associated) vasculitis or systemic lupus erythematosus (SLE), or can be caused by diseases restricted to the kidney, such as membranous nephropathy, IgA nephropathy, and tubulointerstitial nephritis (1). These diseases differ substantially in the primary location of renal tissue damage and in the disease course. The clinical manifestations range from asymptomatic hematuria to nephrotic syndrome with massive urinary protein loss or rapidly progressive glomerulonephritis with irreversible renal failure. Fundamental to each of these diseases is a specific immune reaction with unknown triggers that is followed by an effector phase with destructive inflammation and resulting renal damage. Current treatment options for most patients with immune-mediated glomerular diseases rely on unspecific immunosuppressive agents that are hazardous and only partially efficacious. Despite recent therapeutic advances in this field (2, 3 ), immune-mediated diseases remain a leading cause of end-stage kidney failure worldwide. Thus, determined efforts to characterize underlying immunopathologic mechanisms and to translate results of these studies into treatment concepts are clearly needed for the development of more effective and safer therapies for this group of diseases in the future.

A morphological hallmark of immunemediated kidney diseases is the recruitment of immune cells into the kidney, which correlates with clinical outcome $(4,5)$. In particular, infiltrating effector $\mathrm{T}$ cells of the Th1 and Th17 type are supposed to promote renal

Related Article: https://doi.org/10.1172/JCl142428

Authorship note: CFK, JET, and JHR contributed equally to this work.

Conflict of interest: The authors have declared that no conflict of interest exists.

Copyright: () 2021, American Society for Clinical Investigation.

Reference information: / Clin Invest. 2021;131(12):e150588. https://doi.org/10.1172/JCl150588.

tissue damage through the release of proinflammatory cytokines, ultimately leading to progressive loss of renal function $(5,6)$. A key cytokine for Th17 cell expansion and survival is IL-23, which is composed of two subunits, p19 and p40 (Figure 1A). Of note, the p40 subunit is also part of the Th1 cytokine IL-12. Several experimental and clinical studies have shown that IL-23 promotes $\mathrm{CD} 4^{+} \mathrm{Th} 17$ cell-driven tissue injury in immune-mediated diseases of the skin, joints, CNS, intestine, and kidney (7-10). These studies led to the development of anti-IL-23 p40, and more recently anti-IL-23 p19, monoclonal antibody therapies that have been approved for clinical use in psoriasis and arthritis.

It is generally thought that the IL-23 receptor that mediates the effects of IL-23 is predominantly expressed by lymphocytes and macrophages. In the kidney, dendritic cell-derived IL-23 acts on $\gamma \delta \mathrm{T}$ cells to promote IL-17A-mediated renal inflammation (11) and IL-17A produced by Th17 cells stimulates renal tubular cells to express CXCL5 and CCL20 that recruit neutrophils and augment Th17 cell infiltration (12), respectively.

\section{A mechanism that drives renal tissue injury}

In this issue of the JCI, Li et al. in the Tsokos group identify an additional mechanism of how the IL-23/IL-17 pathway might drive renal tissue injury (13). The authors showed that systemic overexpression of IL-23 directly acted on renal tubular epithelial cells (TECs) to promote an inflammatory microenvironment in the kidney tubulointerstitium (Figure 1B). This mechanism is of great interest, since the tubulointerstitial infiltrate in immune-mediated renal diseases has been reported to play a predominant role in mediating tissue damage (14). On the other hand, this finding is unexpected and in some contrast to studies reporting that elevated IL-23 serum levels in experimental and 
A Classical view of IL-23/IL-17 signaling

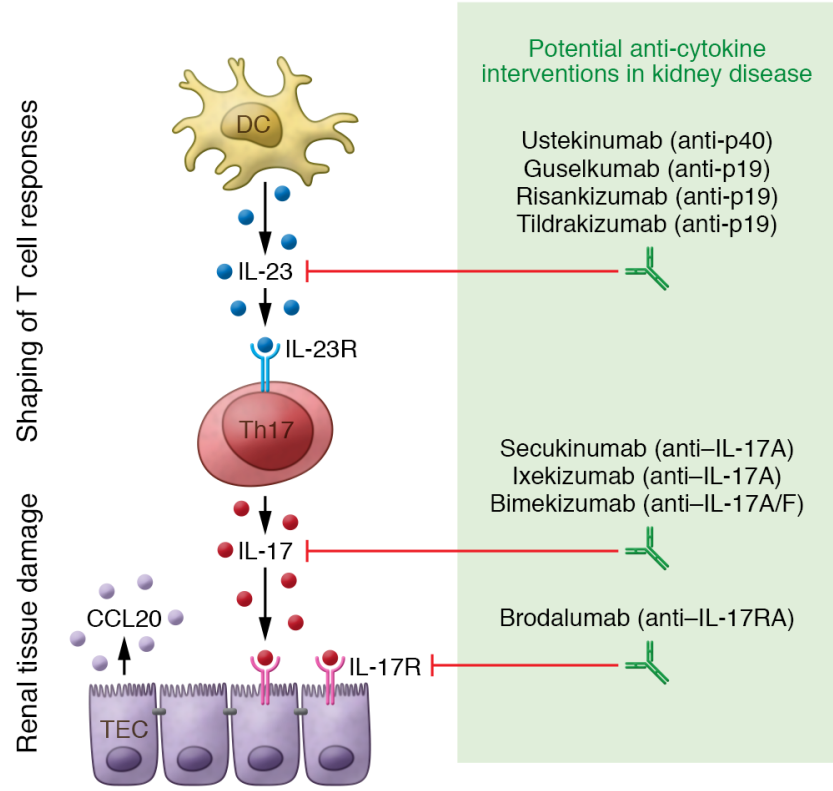

B Direct effects of IL-23 on TECs

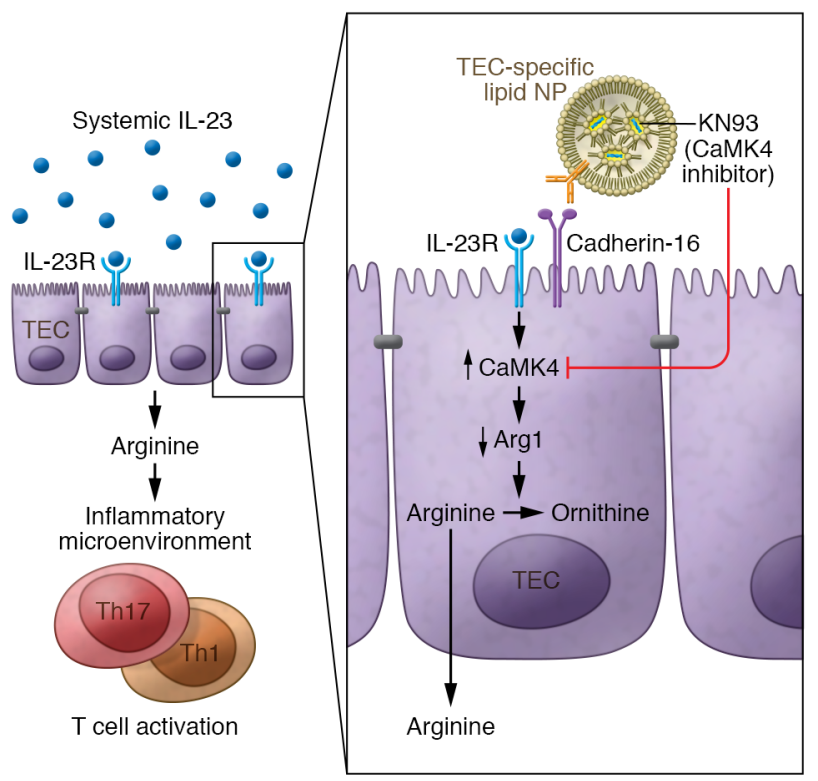

Figure 1. IL-23/IL-17 signaling and T cell activation in immune-mediated kidney disease. (A) In the classical view of inflammatory cytokine signaling, dendritic cell-derived (DC-derived) IL-23 acts on Th17 cells in the local microenvironment of the kidney to maintain and potentiate the Th17 cell immune response. Th17 cells in this way produce IL-17 that triggers CCL20 expression in tubular epithelial cells (TECs) and thereby sustains immune cell infiltration. Several anti-cytokine antibodies already in clinical use target this pathway therapeutically. (B) An alternative mechanism for IL-23-mediated kidney inflammation in which IL-23 directly affects TECs. Li et al. investigated systemic IL-23 overexpression in experimental mouse models. In TECs, IL-23 signaled through the IL-23 receptor (IL-23R) to block arginine turnover, resulting in T cell activation and renal inflammation. The authors used a lipid nanoparticle (NP) system to deliver a CaMK4 inhibitor specifically to TECs. Notably, arginine decreased and fewer T cells infiltrated (13).

human immune-mediated diseases are not associated with kidney inflammation (15). Most cytokines of the IL-23/IL-17 family act locally rather than systemically. Therefore, it can be assumed that the local production of IL-23 also drives renal tissue injury. However, $\mathrm{Li}$ et al. did not investigate the renal expression and cellular source of IL-23 (13). Information about the cellular source of cytokines and the location within the kidney as well as the cellular signal recipients is crucial to decode the cellular cross-talk in immunity. Future studies could address the cellular cytokine source by employing single-cell RNA-sequencing technologies that extend beyond IL-23 and its receptor to also include unsupervised cell-cell interaction analysis covering almost all known possible interactions (16). Noteworthily, these technologies are not restricted to animal models but are already being used in patients with glomerulonephritis (17).

\section{Remodeling the metabolism in inflammatory settings}

Another key finding of Li et al. is that IL-23 directly remodels the metabolism of renal TECs and indirectly that of infiltrating $\mathrm{T}$ cells (13). The supply of energy sources is essential to immune cell metabolism in homeostasis and, all the more important in inflammatory settings, which further intensify competition for limited nutrients (18). Immune cell proliferation and survival, as well as immune cell differentiation and cytokine production, critically depend on nutrient availability, including that of glucose, amino acids, and fatty acids (19). Since the ability to utilize different classes of nutrients is unequally distributed between cells, including immune cells, therapeutic tools to specifically modify nutrient availability in tissues might yield innovative strategies for regulating immune responses. Such strategies that include competitive uptake of nutrients and induction of amino acid-metabolizing enzymes are effectively used by various tumor entities to create microenvironments favoring immunosuppression and immune escape (20). Li and colleagues elegantly dissect such a metabolic mechanism of immunomodulation in the setting of sterile kidney inflammation (13). The authors demonstrate that systemic overexpression of the proinflammatory cytokine IL-23, by inducing the calcium/ calmodulin-dependent protein kinase IV (CaMK4) in proximal TECs (pTECs), is sufficient to locally downregulate the arginine-hydrolyzing enzyme arginase 1 (ARG1). Mechanistically, this systemic overexpression results in increased local arginine levels and promotes an inflammatory milieu with intrarenal $\mathrm{T}$ cell proliferation and subsequent neutrophil influx (Figure 1B). The amino acid arginine has not only emerged as a cornerstone of effector $\mathrm{T}$ cell protein synthesis, enhancing their fitness, activation status, and differentiation, but also as a precursor for multiple metabolites with immunomodulatory properties (21). Intriguingly, in addition to the sophisticated mechanistic experiments in preclinical mouse models, the present study provides evidence that the very same intrarenal metabolic pathway might participate in human autoimmune diseases with kidney involvement, i.e., SLE and ANCAassociated vasculitis.

\section{Clinical implications}

The translational potential of findings in immune-metabolism research is often limited, as targeting of essential metabolic 
pathways with systemically acting pharmacologic compounds in patients will be accompanied by severe side effects. The present study again elegantly circumvents this side-effect caveat by introducing another innovative approach: TEC-specific nanolipogel-based drug delivery (13). In essence, these nanolipogels (nanoscale liposomal polymeric gels; refs. 22, 23) are liposomes loaded with a solubilized small molecule drug bound to a biopolymer matrix. Li et al. used the CaMK4 inhibitor KN93. Cell-specific targeting was achieved by attaching a cell-specific antibody, in this case, anti-cadherin-16 for pTECs, to the outer layer of the liposome (13). But, these liposomes could also be directed against CD4 for $\mathrm{T}$ helper cell targeting (23). This targeting strategy has several advantages over nucleic acid- or cell-based strategies that have been developed in recent years (24), as nanogels are relatively easy to produce, can carry almost any hydrophobic compound, and a large variety of target cell-specific monoclonal antibodies can be attached to their surface by a simple streptavidin-biotin-based adapter system.

In summary, Li et al. confirm the IL-23/ IL-17 pathway as a key driver of immunemediated kidney diseases. A direct IL-23 effect on renal TECs adds another layer of complexity to the standard view of IL-23 as a cytokine that mainly targets lymphocytes. Moreover, $\mathrm{Li}$ and colleagues exploited this mechanism to block the downstream effects of IL-23 cytokine signaling in a cell-specific manner to generate an immune regulatory microenvironment in the kidney and effectively treat renal autoimmune disease in preclinical models
(13). From a translational point of view, the next step in establishing IL-23 targeting as a treatment approach for renal autoimmunity will be to demonstrate clinical benefits of existing IL-23-directed biologicals in proof-of-concept trials with patients.

\section{Acknowledgments}

CFK, JET, and UP acknowledge support from the Deutsche Forschungsgemeinschaft (SFB 1192).

Address correspondence to: Ulf Panzer, Division of Translational Immunology, III. Department of Medicine, University Medical Center Hamburg-Eppendorf, Martinistr. 52, 20246 Hamburg, Germany. Phone: 49.40.7410.51557; Email: panzer@uke.de.

1. Couser WG. Basic and translational concepts of immune-mediated glomerular diseases. J Am Soc Nephrol. 2012;23(3):381-399.

2. Furie R, et al. Two-year, randomized, controlled trial of belimumab in lupus nephritis. $N$ Engl J Med. 2020;383(12):1117-1128.

3. Jayne DRW, et al. Avacopan for the treatment of ANCA-associated vasculitis. $N$ Engl J Med. 2021;384(7):599-609.

4. Kurts C, et al. The immune system and kidney disease: basic concepts and clinical implications. Nat Rev Immunol. 2013;13(10):738-753.

5. Suarez-Fueyo A, et al. T cells and autoimmune kidney disease. Nat Rev Nephrol. 2017;13(6):329-343.

6. Krebs CF, et al. T helper type 17 cells in immune-mediated glomerular disease. Nat Rev Nephrol. 2017;13(10):647-659.

7. Ooi JD, et al. IL-23, not IL-12, directs autoimmunity to the Goodpasture antigen. JAm Soc Nephrol. 2009;20(5):980-989.

8. Paust HJ, et al. The IL-23/Th17 axis contributes to renal injury in experimental glomerulonephritis. J Am Soc Nephrol. 2009;20(5):969-979.

9. Stockinger B, Omenetti S. The dichotomous nature of T helper 17 cells. Nat Rev Immunol.
2017;17(9):535-544.

10. Gaffen SL, et al. The IL-23-IL-17 immune axis: from mechanisms to therapeutic testing. Nat Rev Immunol. 2014;14(9):585-600.

11. Turner JE, et al. IL-17A production by renal $\gamma \delta \mathrm{T}$ cells promotes kidney injury in crescentic GN. JAm Soc Nephrol. 2012;23(9):1486-1495.

12. Disteldorf EM, et al. CXCL5 drives neutrophil recruitment in TH17-mediated GN. J Am Soc Nephrol. 2015;26(1):55-66.

13. Li H, et al. IL-23 reshapes kidney resident cell metabolism and promotes local kidney inflammation. JClin Invest. 2021;131(12):e142428.

14. Bohle A, et al. On the pathogenesis of chronic renal failure in primary glomerulopathies: a view from the interstitium. Exp Nephrol. 1994;2(4):205-210.

15. Sherlock JP, et al. IL-23 induces spondyloarthropathy by acting on $\mathrm{ROR}-\gamma \mathrm{t}^{+} \mathrm{CD}^{+} \mathrm{CD} 4$ CD8- entheseal resident T cells. Nat Med. 2012;18(7):1069-1076

16. Giladi A, Amit I. Single-cell genomics: a stepping stone for future immunology discoveries. Cell. 2018;172(1-2):14-21.

17. Krebs CF, et al. Pathogen-induced tissue-resident memory $\mathrm{T}_{\mathrm{H}} 17\left(\mathrm{~T}_{\mathrm{RM}} 17\right)$ cells amplify autoimmune kidney disease. Sci Immunol. 2020;5(50):eaba4163.

18. Jung J, et al. Metabolism as a guiding force for immunity. Nat Cell Biol. 2019;21(1):85-93.

19. O'Neill LA, et al. A guide to immunometabolism for immunologists. Nat Rev Immunol. 2016;16(9):553-565.

20. Kedia-Mehta N, Finlay DK. Competition for nutrients and its role in controlling immune responses. Nat Commun. 2019;10(1):2123.

21. Geiger R, et al. L-Arginine modulates T cell metabolism and enhances survival and antitumor activity. Cell. 2016;167(3):829-842.

22. Park J, et al. Combination delivery of TGF- $\beta$ inhibitor and IL- 2 by nanoscale liposomal polymeric gels enhances tumour immunotherapy. Nat Mater. 2012;11(10):895-905.

23. Look M, et al. Nanogel-based delivery of mycophenolic acid ameliorates systemic lupus erythematosus in mice. JClin Invest. 2013;123(4):1741-1749.

24. Zhao Z, et al. Targeting strategies for tissue-specific drug delivery. Cell. 2020;181(1):151-167. 